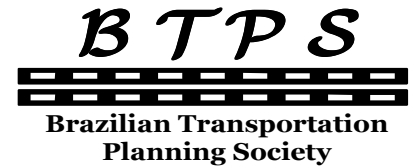

Planning Society
Journal of Transport Literature

Vol. 7, n. 4, pp. 75-106, Oct. 2013

Research Directory

\section{JTL|RELIT}

www.transport-literature.org ISSN 2238-1031

\title{
Avaliação da eficiência dos portos utilizando análise envoltória de dados: estudo de caso dos portos da região nordeste do Brasil
}

[Performance evaluation of ports using data envelopment analysis: a case study on the ports of Northeast region of Brazil]

José Nauri C. Sousa Júnior, Ernesto F. Nobre Júnior, Bruno de Athayde Prata*, João Carlos C. B. Soares de Mello

Universidade Federal do Ceará, Universidade Federal do Ceará,

Universidade Federal do Ceará, Universidade Federal Fluminense

Submitted 1 Sep 2012; received in revised form 28 Jan 2013; accepted 28 Jan 2013

\section{Resumo}

O sistema portuário é elemento-chave para o desenvolvimento macroeconômico da sua área de influência. A avaliação de desempenho do setor portuário torna-se fundamental para o monitoramento e o aprimoramento das atividades deste setor para buscar atingir a eficiência. Para que se possa procurar a eficiência é preciso medi-la e conhecer as ineficiências. Estas medidas podem ser feitas com a Análise Envoltória de Dados (Data Envelopment Analysis - DEA) que é um método baseado em programação matemática, que analisa, com base nos recursos (inputs) e produtos (outputs) utilizados em dado processo, a eficiência de um conjunto de unidades tomadoras de decisão (Decision Making Units - DMU). 0 presente trabalho teve como objetivo elaborar e analisar modelo para a medição da eficiência dos portos da Região Nordeste do Brasil, no ano de 2006, adotando o método DEA. A seleção de variáveis foi feita com auxílio do método Compensatório de Normalização Única, o qual determina as variáveis a serem utilizadas através do índice $S$ que depende da eficiência média (relação causal) e da quantidade de DMU na fronteira (poder de discriminação). Foram analisadas as instalações e operações de contêineres e granéis sólidos dos principais portos da Região Nordeste. A obtenção da eficiência portuária, efetuada por tipo de carga, auxilia no planejamento de médio e longo prazo, de forma a organizar os portos para aumentar a movimentação horária e a movimentação de cargas, contribuindo, assim, para a eficiência dos terminais.

Palavras-Chave: planejamento portuário, análise envoltória de dados, avaliação de desempenho.

\begin{abstract}
The port system is a key element for the macroeconomic development of its area of influence. The performance evaluation of the port sector becomes fundamental to the monitoring and improvement of the activities of this sector aiming at accomplishing efficiency. The Data Envelopment Analysis - DEA is a method based on mathematic programming which analyzes, based on the inputs and outputs utilized in a certain process, the efficiency of a set of Decision Making Units - DMU. This paper aims to elaborate and analyze models for the efficiency measuring of the ports in Brazil's northwest in 2006, adopting the DEA method. The variables selection was done with the aid of the Compensatory method of Normalization (Single Parameter), which determines the variables to be utilized through index $\mathrm{S}$ that depends on the average efficiency (causal relation) and the quantity of DMU in the frontier (discrimination power). The facilities of containers and solid bulks of the main ports in northwest were analyzed. The obtaining of the port efficiency executed by the type of load helps in the medium and long-term planning, so that it organizes the ports to increase the timetable turnover and the loads turnover, contributing to the efficiency of the terminals.
\end{abstract}

Key words: port planning, data envelopment analysis, performance evaluation.

*Email: baprata@ufc.br.

\section{Recommended Citation}

Sousa Jr., J. N. C., Nobre Jr., E. F., Prata, B. A. and Mello, J. C. C. B. S. (2013) Avaliação da eficiência dos portos utilizando análise envoltória de dados: estudo de caso dos portos da região nordeste do Brasil. Journal of Transport Literature, vol. 7, n. 4, pp. 75106.

- JTL/RELIT is a fully electronic, peer-reviewed, open access, international journal focused on emerging transport markets and published by BPTS - Brazilian Transport Planning Society. Website www.transport-literature.org. ISSN 2238-1031. 


\section{Introdução}

O sistema de transporte, juntamente com as infraestruturas correlatas, mostra-se fundamental para o desempenho satisfatório e o crescimento do mercado. Segundo Rodrigues (2007), um sistema de transportes é constituído pelo modo (rodoviário, aquaviário, ferroviário, dutoviário e aeroviário), pela forma (relacionamento entre os vários modos de transportes), pelo meio (elemento transportador) e pelas instalações complementares (terminais de carga).

Segundo Caixeta Filho (2001), os transportes têm a função básica de proporcionar elevação na disponibilidade de bens, pois colocam as mercadorias em locais onde não estariam disponíveis. O transporte tem o importante papel de tentar romper barreiras, provocadas pelo isolamento geográfico, permitindo o escoamento da produção e a comercialização de produtos. O sistema portuário faz parte do sistema de transporte, com a função de deslocar pessoas ou cargas.

Collyer (2008) define porto como fronteira nacional aberta, entreposto dinâmico de mercadorias, em que se realizam atividades (aduaneiras, alfandegárias, comerciais, sanitárias, tributárias, imigratórias etc.). É o portão de entrada e saída de riquezas, local de abrigo das embarcações, fonte de suprimento das atividades offshore, ponto estratégico de segurança das nações e, sobretudo, um importante elo da cadeia logística mundial.

A estruturação dos portos brasileiros é bastante fragmentada, pois não foi concebida de forma integrada. Na concepção dos portos, não houve planejamento integrado, de forma que os portos foram instalando-se em regiões e localidades sem o devido entendimento, para o país, de sua importância.

Além dessa falta de planejamento integrado, os portos brasileiros são precários devido à falta de indicadores confiáveis capazes de analisar a situação atual e o planejamento em médio e longo prazo. A ausência de dados ou a utilização de dados não confiáveis prejudica a análise, podendo até inviabilizar o planejamento do setor.

De acordo com Uderman et al. (2012), um sistema portuário ineficiente e caro implica custos adicionais significativos para uma série de empreendimentos produtivos, engendrando, como 
consequência direta, um ambiente pouco propício ao crescimento do nível de atividade econômica e à atração de novos investimentos.

Conforme Collyer (2013), no Brasil a Logística tem deixado a desejar, pois apesar do expressivo aumento da produção agrícola e das obras de infraestrutura de transportes, estes não vêm sendo acompanhados de uma regulação do setor de transportes que possa fazer face a essa demanda de forma eficiente.

Portanto, o setor necessita de um diagnóstico de sua da situação atual, a fim de, em médio prazo, realizar um planejamento integrado, visando o incremento da qualidade do serviço prestado e uma melhor alocação dos recursos empregados.

Cortez et al. (2013), percebe a necessidade do governo em aprimorar a infraestrutura provida de modo a acabar com o gargalo logístico existente no entorno dos portos, aumentando assim o grau de competitividade da indústria nacional.

Este artigo tem como objetivo determinar e analisar a eficiência, através do método Análise Envoltória de Dados (Data Envelopment Analysis - DEA), dos portos da Região Nordeste do Brasil.

O artigo é composto por outras três seções, descritas a seguir. Na segunda seção, é apresentado o método DEA. Na terceira seção, é apresentado o estudo de caso referente aos portos da Região Nordeste do Brasil, referente a portos que operam contêineres e granéis sólidos. Por fim, na quarta seção, são apresentadas as conclusões do estudo.

\section{Análise envoltória de dados}

A Análise Envoltória de Dados - DEA é um método baseado em programação linear, cujo objetivo é medir a eficiência de entidades denominadas de unidades tomadoras de decisão (Decision Making Units - DMU). Tal medição é realizada de maneira comparativa, tendo como base as informações dos recursos (inputs) e dos produtos (outputs) de cada DMU.

Este método foi formulado e desenvolvido por Charnes, Cooper e Rhodes (1978), baseado em programação matemática em que a medida de eficiência é obtida pela razão da soma ponderada dos dados de saída (outputs) pelos de entrada (inputs). 
Thanassoulis et al.(1996) entendem DEA como abordagem não-paramétrica para estimar o máximo de produção para os dados de entrada (inputs) ou níveis mínimos dos dados de entrada para os dados de produção (outputs).

Lins e Angulo Meza (2000) afirmam que DEA é o método para apoio à decisão de natureza multicritério, que procura modelar a complexidade do mundo real. Conforme Soares de Mello et al. (2003), o objetivo da Análise Envoltória de Dados é comparar determinado número de DMU que realizam tarefas semelhantes, diferenciando-se nas quantidades de inputs que consomem e de outputs que produzem.

Cullinane et al. (2004) afirmam que DEA pode ser definida como método não-paramétrico para a mensuração da eficiência de DMU com múltiplos dados de entradas (inputs) e/ou de saídas (outputs). Cook e Zhu (2005) definem DEA como abordagem para avaliar o desempenho de conjunto de entidades que convertem as várias entradas (inputs) para várias saídas (outputs).

Os métodos não-paramétricos são conhecidos por realizarem diversas análises, de forma relativamente simples, pelo fato de não precisarem transformar os dados em indicador-padrão, como acontece nos métodos paramétricos.

\subsection{Seleção de variáveis}

Um ponto crítico na modelagem DEA é a seleção de variáveis. Uma melhor explicação do problema estudado pode requerer um grande número de variáveis. Entretanto, as características matemáticas de DEA podem fazer com que modelos com um elevado número de variáveis avaliem como eficientes um grande número de DMU, o que tira a utilidade do modelo como ferramenta de avaliação. Thanassoulis (1996) afirma que a alteração do conjunto de variáveis selecionadas poderá ter grande impacto no resultado da obtenção da eficiência das DMU. Portanto, a etapa de seleção das variáveis é fundamental para o refinamento do método DEA.

A seleção de variáveis escolhe as que são relevantes para estabelecer a eficiência das DMU selecionadas. A definição de inputs e outputs pode ser baseada no conhecimento de especialistas, bem como por algum método estatístico ou multicritério. 
A apresentação detalhada de alguns métodos de seleção de variáveis pode ser visto em Senra et al. (2007) e Angulo Meza et al. (2007), que analisam cinco métodos de seleção: I-O Stepwise, Multicritério, Multicritério Combinatório Inicial (MCI), Multicritério Combinatório Parcial (MCP) e o Compensatório de Normalização Única (CNU).

O método de seleção utilizado neste trabalho foi o CNU, que, conforme Senra et al. (2007), é um método aconselhado para descartar variáveis que agregam pouca ou nenhuma eficiência média ao modelo (menos representativas) e destacar as variáveis que agregam maior eficiência média (mais representativas).

\subsection{Método Compensatório de Normalização Única}

O método CNU foi proposto por Angulo Meza et al. (2007), sendo adaptado do método multicritério combinatório por cenários. Ao contrário do método por cenários, a normalização não considera os valores máximos e mínimos efetivamente atingidos para a eficiência e o número de DMU na fronteira para cada quantidade de variáveis, o método de normalização leva em consideração os valores extremos teoricamente alcançáveis (ANGULO MEZA et al. 2007).

O método de normalização considera a eficiência média normalizada $\left(S_{E F}\right)$ que varia de 0 (eficiência mínima) a 10 (eficiência máxima). $O S_{E F}$ é calculado pela seguinte expressão $S_{E F}=\frac{\text { Eficiência média }}{10}$. O poder de discriminação normalizado $\left(S_{D I S}\right)$ apresenta valor de 0 (maior número de DMU eficientes) a 10 (menor número de DMU eficientes). O $S_{D I S}$ é calculado pela seguinte expressão $S_{D I S}=10 \frac{n-N}{n-1}$, onde $n$ é o número total de DMU e $N$ é o número total de DMU eficientes.

Aplica-se esta normalização a cada etapa do método para escolher a próxima variável a ser inserida no modelo. O conjunto final de variáveis a ser utilizado é o que apresentar maior índice $S=S_{E F}+S_{D I S}$, que é medida de compromisso entre o bom ajuste à fronteira e a boa discriminação do modelo. 


\subsection{Estruturação do modelo DEA CCR}

Definidas as variáveis a serem utilizadas, formaliza-se o método DEA. A estruturação e representação do modelo CCR será apresentado a seguir.

O modelo CCR pode ser estruturado supondo $n$ DMU utilizando $I$ inputs para produzir $O$ outputs. O CCR pode ser orientado a input ou a output. As formulações, apresentadas a seguir, são baseadas em Talluri (2000).

O problema de programação linear do modelo CCR orientado a inputs tem a seguinte estrutura:

$$
\operatorname{Maximizar} \quad z_{0}=\sum_{y=1}^{O} v_{y} o_{y 0}
$$

Sujeito a:

$$
\begin{gathered}
\sum_{x=1}^{I} u_{x} I_{x 0}=1 \\
\sum_{y=1}^{O} v_{y} o_{y k}-\sum_{x=1}^{I} u_{x} I_{x k} \leq 0, \forall k \\
u_{x}, v_{y} \geq 0, \forall x, y
\end{gathered}
$$

em que,

$z_{0}$ : Eficiência da DMU 0;

I: número total de inputs;

$O:$ número total de outputs;

$n$ : número total de DMU;

$I_{x k}$ : quantidade de input $x$ para DMU $k ; k=1,2, \ldots, n$;

$O_{y k}$ : quantidade de output y para DMU $k ; k=1,2, \ldots, n$;

$u_{x}$ : peso outorgado ao input $x$;

$v_{y}$ : peso outorgado ao output $y$.

A equação (1) consiste na função objetivo do modelo e representa a eficiência da DMU, a qual será maximizada. A equação (2) representa a restrição de que o somatório do produto dos inputs pelo peso outorgado será igual a 1 . O conjunto de equações do tipo (3) garante que a subtração do somatório dos outputs pelo somatório dos inputs seja menor ou igual a 0 . $\mathrm{O}$ conjunto de equações do tipo (4) determina que os pesos, tanto dos inputs quanto dos outputs, sejam não-negativos para qualquer valor de $x$ e $y$. 
Conforme Soares de Mello et al. (2003), a estrutura matemática desse modelo permite que uma DMU seja considerada eficiente com várias combinações de pesos. Caso um dos pesos seja zero, significa que a variável foi desconsiderada.

Coelli (1999) sugere que a decisão da orientação do modelo a ser utilizado (input ou output) deve ser feita com a análise das variáveis selecionadas, de modo que a orientação seja feita com os dados mais confiáveis.

O método DEA vem sendo utilizado, nos últimos anos, em várias áreas. Na Tabela 1, são apresentados alguns trabalhos com aplicação de DEA em estudos voltados para o setor portuário.

Pelos estudos apresentados na Tabela 1, há a utilização dos dois modelos DEA (CCR e BCC) na análise da eficiência portuária. O estudo adotará o modelo CCR, pois o uso do BCC iria agravar a relação entre variáveis e DMU, ou seja, a eficiência obtida não apresentaria resultado que reflita a situação dos terminais portuários.

Conforme afirma Soares de Mello (2004), o modelo BCC atribuiria eficiência de 100\% para unidade com menor input e maior output, independente da relação entre eles.

$\mathrm{Na}$ análise dos portos, poderia ter um terminal eficiente apenas porque é de porte muito grande, sem considerar uma relação com as demais variáveis.

No que se refere à análise de eficiência de portos, um problema que surge é a heterogeneidade dos terminais, devido às suas características operacionais. Comparar a eficiência de portos especializados em diferentes tipos de carga pode levar a análises equivocadas. Conforme Bertoloto (2010), o conjunto de DMU deve ser homogêneo, para que se faça uso do método DEA.

Dyson et al. (2001) definem como homogêneas as DMU que contemplem os seguintes aspectos: (i) produzam produtos ou ofertem serviços similares, de forma que possuam inputs e outputs comparáveis; (ii) possuam o mesmo conjunto de recursos disponíveis; e (iii) operem em ambientes similares. 
Tabela 1 - Síntese dos trabalhos de DEA no setor portuário.

\begin{tabular}{|c|c|c|c|}
\hline AUTORES & $\begin{array}{l}\text { MODELO } \\
\text { DEA }\end{array}$ & INPUTS & OUTPUTS \\
\hline Roll e Hayuth (1993) & $\mathrm{CCR}$ & $\begin{array}{l}\text { - Capital; } \\
\text { - } \mathrm{N}^{\mathrm{o}} \text { de funcionários } \\
\text { - Tipo de carga }\end{array}$ & $\begin{array}{l}\text { - Nível de serviço } \\
\text { - Movimentação de carga } \\
\text { - Satisfação dos usuários } \\
\text { - } \mathrm{N}^{\circ} \text { de atracações } \\
\end{array}$ \\
\hline $\begin{array}{l}\text { Martinez-Budria et al. } \\
\text { (1999) }\end{array}$ & $\mathrm{BCC}$ & $\begin{array}{l}\text { - Despesas com pessoal } \\
\text { - Taxas de depreciação } \\
\text { - Outros gastos }\end{array}$ & $\begin{array}{ll}\text { - } & \text { Total de carga } \\
\text { movimentada } \\
\text { - Receita obtida no aluguel } \\
\text { de facilidades } \\
\end{array}$ \\
\hline Tongzon (2001) & $\mathrm{CCR}$ & $\begin{array}{l}\text { - } \mathrm{N}^{\circ} \text { de guindastes } \\
\text { - } \mathrm{N}^{\mathrm{o}} \text { de berços } \\
\text { - } \mathrm{N}^{\mathrm{o}} \text { de rebocadores } \\
\text { - } \mathrm{N}^{\mathrm{o}} \text { de funcionários } \\
\text { - Área do terminal } \\
\text { - Tempo de espera } \\
\end{array}$ & - TEU \\
\hline Vallentine e Gray (2001) & $\mathrm{CCR}$ & $\begin{array}{l}\text { - Tamanho do berço } \\
\text { - Investimento }\end{array}$ & $\begin{array}{l}\text { - } \mathrm{N}^{\circ} \text { de contêineres } \\
\text { - Total de toneladas } \\
\text { movimentadas }\end{array}$ \\
\hline Itoh (2002) & CCR e BCC & $\begin{array}{l}\text { - Área do terminal } \\
\text { - } \mathrm{N}^{\circ} \text { de berços } \\
\text { - } \mathrm{N}^{\mathrm{o}} \text { de funcionários } \\
\text { - } \mathrm{N}^{\mathrm{o}} \text { de guindastes } \\
\end{array}$ & - TEU \\
\hline Serrano e Castellano (2003) & $\mathrm{BCC}$ & $\begin{array}{l}\text { - Tamanho do berço } \\
\text { - Área do terminal } \\
\text { - } \mathrm{N}^{\circ} \text { de guindastes }\end{array}$ & $\begin{array}{l}\text { - TEU } \\
\text { - Toneladas movimentadas }\end{array}$ \\
\hline Turner et al. (2004) & - & $\begin{array}{l}\text { - Tamanho do berço } \\
\text { - Área do terminal } \\
\text { - } \mathrm{N}^{\circ} \text { de guindastes } \\
\end{array}$ & - TEU \\
\hline Cullinane et al. (2004) & CCR e BCC & $\begin{array}{l}\text { - Tamanho do berço } \\
\text { - Área do terminal } \\
\text { - } \mathrm{N}^{\circ} \text { de guindastes de berço } \\
\text { - } \mathrm{N}^{\circ} \text { de guindastes de pátio } \\
\text { - } \mathrm{N}^{\circ} \text { de Straddle Carrier } \\
\end{array}$ & - TEU \\
\hline Rios (2006) & $\mathrm{CCR}$ e $\mathrm{BCC}$ & $\begin{array}{l}\text { - } \mathrm{N}^{\circ} \text { de guindastes } \\
\text { - } \mathrm{N}^{\mathrm{o}} \text { de berço } \\
\text { - Área do terminal } \\
\text { - } \mathrm{N}^{\mathrm{o}} \text { de funcionários } \\
\text { - } \mathrm{N}^{\mathrm{o}} \text { de equipamentos }\end{array}$ & $\begin{array}{l}\text { - } \text { TEU } \\
\text { Prancha média de } \\
\text { movimentação de } \\
\text { contêineres por hora }\end{array}$ \\
\hline Fontes (2006) & $\mathrm{BCC}$ & $\begin{array}{l}\text { - Extensão total do cais } \\
\text { acostável }\end{array}$ & $\begin{array}{l}\text { - Movimentação total de } \\
\text { embarcações } \\
\text { - Movimentação total da } \\
\text { carga movimentada }\end{array}$ \\
\hline Sousa Júnior (2008) & CCR & $\begin{array}{l}\text { - Comprimento dos berços } \\
\text { - Calado admissível }\end{array}$ & $\begin{array}{l}\text { Movimentação (em } \\
\text { toneladas ou em número } \\
\text { de contêineres) }\end{array}$ \\
\hline
\end{tabular}


Deste modo, a análise de eficiência realizada neste artigo optou pelo agrupamento das DMU em dois clusters homogêneos: portos que operam contêineres e portos que operam granéis sólidos.

\section{Estudo de Caso}

\subsection{Considerações iniciais}

Os principais portos e terminais da Região Nordeste do Brasil são analisados neste trabalho. O estudo abrange 16 portos e terminais, sendo 11 portos organizados e geridos por empresas públicas, e 5 terminais administrados por empresas privadas.

A seguir, é apresentada a definição das variáveis utilizadas no estudo. A escolha das variáveis deu-se em função de estudos anteriores e das informações obtidas junto aos portos.

A definição propicia o entendimento do significado da variável, além de poder realizar a análise do setor portuário com conhecimento de suas principais características.

As variáveis estão divididas em: variáveis de infraestrutura (itens de $i$ a iii) e de operação (itens de iv a $v i)$.

\section{Variáveis de Infraestrutura}

i. Calado (em m): designação dada à profundidade para a qual se encontra o ponto mais baixo da quilha do navio;

ii. Extensão do berço (em $\mathrm{m}$ ): medida de comprimento do local de atracação ou desatracação do navio no porto;

iii. Capacidade estática (em t, TEU -Twenty-feet Equivalent Unit - ou em unidade de área): capacidade de armazenamento do porto por tipo de carga;

\section{Variáveis de Operação}

iv. Navios (unidade): Por tipo - carga geral, graneleiro, de contêineres, roll on roll off, de porão refrigerado (reefer), etc. (ANTAq, 2003);

v. Movimentação (em $\mathrm{t}$ ou TEU): medida em unidades ou em TEU por determinado período de tempo (ANTAq, 2003); 
vi. Movimentação horária ( $\mathrm{t} / \mathrm{dia}$ ou TEU/h): Conhecida no setor portuário como produtividade. É a quantidade de carga movimentada por navio durante o seu atendimento no berço, dividido pelo tempo que este passou atracado no berço (em horas) (ANTAq, 2003).

A coleta das variáveis ocorreu junto aos portos, de forma a evitar distorções de fontes secundárias (órgãos do setor portuário). Os dados operacionais foram de 2006, por ser o único ano obtido para todos os portos em análise.

\subsection{Caso de estudo 1: avaliação dos terminais de contêineres}

A Tabela 2 mostra os dados dos portos que operam contêineres. As variáveis de inputs são relacionadas à infraestrutura; os outputs são referentes à operação anual (no estudo o ano analisado é o de 2006).

Tabela 2 - Variáveis das DMU que movimentam contêiner.

\begin{tabular}{ccccccc}
\hline Variáveis & Salvador & Suape & Pecém & Fortaleza & Natal & Maceió \\
\hline Calado (m) & 12 & 12,50 & 14 & 10,50 & 10 & 10,50 \\
\hline $\begin{array}{l}\text { Extensão do berço (m) } \\
\text { Capacidade estática (TEU) }\end{array}$ & 661 & 935 & 700 & 690 & 400 & 400 \\
\hline \begin{tabular}{l} 
Movios (qde) \\
\hline Movimentação (TEU)
\end{tabular} & 615 & 474 & 308 & 177 & 31 & 24 \\
\hline
\end{tabular}

Com os dados obtidos dos portos, a próxima etapa foi à seleção das variáveis, de modo a selecionar os dados que pudessem representar as principais características físicas e operacionais do porto, e, assim, obter a sua eficiência. Essa etapa é apresentada na seção a seguir 


\section{Seleção de variáveis}

A seleção de variáveis por tipo de carga (contêiner) adotada consistiu no uso do Método Compensatório de Normalização Única (CNU), consideradas no modelo CCR. A etapa do método de seleção Compensatório de Normalização Única (CNU) consistiu em calcular o $S$ de cada par de input-output possível.

Na etapa inicial para contêineres, foram calculados nove pares input-output. As Tabelas 3, 4 e 5 apresentam as eficiências obtidas de cada DMU, a eficiência média e os índices $S_{E F}, S_{D I S}$ e $S$ para cada par de variáveis utilizadas.

\section{Tabela 3 - Seleção de variáveis para contêineres etapa 1 com input berço.}

\begin{tabular}{|c|c|c|c|}
\hline Input & $\operatorname{Berço~(m)~}$ & $\operatorname{Berço~(m)~}$ & $\operatorname{Berço~(m)~}$ \\
\hline Output & Navios & Movimentação & Movimentação horária \\
\hline Salvador & 1,00 & 1,00 & 1,00 \\
\hline Suape & 0,54 & 0,61 & 0,68 \\
\hline Pecém & 0,47 & 0,47 & 0,78 \\
\hline Fortaleza & 0,28 & 0,24 & 0,46 \\
\hline Natal & 0,08 & 0,04 & 0,86 \\
\hline Maceió & 0,06 & 0,06 & 0,86 \\
\hline Eficiência média & 0,41 & 0,40 & 0,77 \\
\hline$S_{E F}$ & 4,07 & 4,03 & 7,73 \\
\hline $\mathrm{N}^{\circ}$ DMU eficientes & 1,00 & 1,00 & 1,00 \\
\hline$S_{D I S}$ & 10 & 10 & 10 \\
\hline$S$ & 14,069 & 14,033 & 17,731 \\
\hline
\end{tabular}

O par input-output que apresentou maior índice $S$, nessa primeira etapa, foi composto pelas variáveis "berço" e "movimentação horária", com valor de 17,731. Além de apresentar maior índice $S$, também obteve o maior índice de eficiência média $\left(S_{E F}\right)$ igual a 7,73.

Na segunda etapa, adicionou-se mais uma variável ao par "berço" e "movimentação horária".

Para essa etapa, foram calculados quatro cenários, encontrando-se o maior índice de eficiência média $\left(S_{E F}\right)$ 8,28 com a adição da variável "calado", o que representa a melhor relação causal. Porém, a adição da variável "capacidade" também obteve $S_{E F}$ próximo ao maior valor obtido, sendo igual a 8,15. O maior índice $S$ obtido foi 18,282 para o cenário que continha a variável "calado".

Na terceira etapa, incluiu-se mais uma variável, sendo gerados três cenários. A Tabela 4 apresenta a eficiência média e os índices $S_{E F}, S_{D I S}$ e $S$. 


\section{Tabela 4 - Seleção de variáveis para contêineres etapa 3 com "berço", "calado" e "movimentação horária".}

\begin{tabular}{l|c|c|c}
\hline Input & Capacidade & - & - \\
\hline Output & - & Navios & Movimentação \\
\hline Salvador & 1,000 & 1,000 & 1,000 \\
\hline Suape & 0,918 & 0,918 & 0,820 \\
\hline Pecém & 0,780 & 0,780 & 0,780 \\
\hline Fortaleza & 0,547 & 0,547 & 0,547 \\
\hline Natal & 1,000 & 0,862 & 0,862 \\
\hline Maceió & 0,975 & 0,862 & 0,862 \\
\hline Eficiência média & 0,870 & 0,828 & 0,828 \\
\hline$S_{E F}$ & 8,70 & 8,28 & 8,28 \\
\hline $\mathrm{N}^{\circ}$ DMU eficientes & 2,00 & 1,00 & 1,00 \\
\hline$S_{D I S}$ & 8,00 & 10,00 & 10,00 \\
\hline$S$ & 16,70 & 18,282 & 18,282 \\
\hline
\end{tabular}

A maior relação causal $\left(S_{E F}\right)$ obtida na Tabela 4 foi com a adição da variável "capacidade", resultando no valor 8,70 .

Na terceira etapa, foram três os índices $S$ obtidos (Tabela 4): dois para o valor de 18,282 com as variáveis "navios" e "movimentação" e um para o valor de 16,70 com a variável "capacidade". Portanto, nesta etapa, os cenários com as variáveis "navios" e "movimentação" obtiveram o mesmo valor de índice $S$ da segunda etapa $(18,282)$ com a adição da variável "calado".

A Tabela 5 apresenta a quantidade de cenários gerados em cada etapa, as variáveis do cenário selecionado e o valor do maior índice $S$ de cada etapa.

Tabela 5 - Etapas do Método CNU para contêiner.

\begin{tabular}{c|c|l|r}
\hline Etapas & Cenários & \multicolumn{1}{|c|}{ Variáveis } & \multicolumn{2}{c}{ Índice $S$} \\
\hline 1 & 9 & $\begin{array}{l}\text { Berço } \\
\text { Movimentação horária }\end{array}$ & 17,731 \\
\hline 2 & 4 & Calado & 18,282 \\
\hline 3 & 3 & $\begin{array}{l}\text { Navios } \\
\text { Movimentação }\end{array}$ & 18,282 \\
\hline
\end{tabular}

Como se pode notar na Tabela 5 não houve acréscimo do índice $S$ da segunda para a terceira etapa. Serão analisados os modelos com 2, 3 e 4 variáveis, composto pelas seguintes variáveis: "berço" (input), “calado" (input), "Movimentação horária” (output), "movimentação" (output) e "navios" (output). 
A etapa de seleção de variáveis é concluída com a determinação dos dados a serem utilizados na aplicação dos modelos DEA, a aplicação do modelo a ser utilizado tem que ser o mesmo modelo utilizado na seleção de variáveis.

A seção a seguir apresenta a aplicação dos modelos DEA/CCR.

\section{Análise dos resultados}

A aplicação do método DEA foi realizada por meio do SIAD - Sistema Integrado de Apoio à Decisão, desenvolvido por Angulo Meza et al. (2005).

A versão utilizada é o SIADv3.0. O programa permite utilizar até 100 DMU e 20 variáveis equilibradas entre inputs e outputs. Com a utilização do programa (SIAD), é obtida agilidade na determinação das eficiências, tendo em vista que possui interface de fácil manuseio por parte do usuário. O SIAD já foi utilizado em outros trabalhos acadêmicos, tendo propiciado resultados satisfatórios com o método DEA, o que justifica seu emprego no presente estudo.

A análise da eficiência aos portos de contêiner foi realizada com modelo o CCR orientado a input e output para julgar os resultados obtidos.

Os cenários obtidos foram de acordo com as variáveis selecionadas no método de seleção CNU, apresentado na Tabela 5. As eficiências obtidas para a avaliação das DMU que operam contêineres foram analisadas de acordo com quatro cenários, apresentados na Tabela 6.

Tabela 6 - Etapas do Método de seleção CNU para contêiner.

\begin{tabular}{c|c|c|c|c}
\hline CENÁRIO & 1 & 2 & 3 & 4 \\
\hline & Berço & Berço & Berço & Berço \\
& Movimentação & Calado & Calado \\
VARIÁVEIS & horária & Movimentação & horária \\
& & & horária \\
& & Navios & $\begin{array}{c}\text { Movimentação } \\
\text { horária } \\
\text { Movimentação }\end{array}$ \\
\hline
\end{tabular}


Os resultados do “cenário 1”, para o modelo DEA/CCR, constam na Tabela 7.

Tabela 7 - Eficiência dos portos de contêineres.

\begin{tabular}{l|r|r|r|r}
\hline & Cenário 1 & Cenário 2 & Cenário 3 & Cenário 4 \\
\hline Salvador & 1,00 & 1,00 & 1,00 & 1,00 \\
\hline Suape & 0,68 & 0,92 & 0,92 & 0,92 \\
\hline Pecém & 0,78 & 0,78 & 0,78 & 0,78 \\
\hline Fortaleza & 0,46 & 0,55 & 0,55 & 0,55 \\
\hline Natal & 0,86 & 0,86 & 0,86 & 0,86 \\
\hline Maceió & 0,86 & 0,86 & 0,86 & 0,86 \\
\hline
\end{tabular}

O único porto eficiente nos quatros cenários foi o porto de Salvador e mais ineficiente foi o porto de Fortaleza.

Os resultados dos modelos, apresentados na Tabela 7, foram iguais nos cenários 2, 3 e 4, ou seja, nesse caso, não afetará a análise a adição das variáveis "navios" ou "movimentação".

O "cenário 1" apresentou resultados diferentes do "cenário 2" para os portos de: Suape, Fortaleza, Natal e Maceió, ou seja, a adição da variável "calado" não altera o índice de eficiência dos portos: Pecém e Salvador.

Além da análise do índice de eficiência, é importante a verificação dos pesos fornecidos pelo modelo a cada variável.

A Tabela 8 apresenta a média dos pesos das variáveis dos modelos DEA/CCR orientados a input.

A variável com maior importância na obtenção do índice de eficiência foi "movimentação horária", seguida da variável "calado". A variável "navios" teve valor de peso zero e quase zero, ou seja, o modelo praticamente desconsiderou essa variável para que as DMU (portos) obtivessem a eficiência máxima possível.

Tabela 8 - Média dos pesos das variáveis dos modelos DEA/CCR orientado a input.

\begin{tabular}{l|c|c|c|c}
\hline \multicolumn{1}{c|}{ Variáveis } & Cenário 1 & Cenário 2 & Cenário 3 & Cenário 4 \\
\hline Berço & 0,0017 & 0,0013 & 0,0013 & 0,0013 \\
\hline Movimentação horária & 0,0501 & 0,0533 & 0,0460 & 0,0460 \\
\hline Calado & - & 0,0292 & 0,0292 & 0,0292 \\
\hline Navios & - & - & 0,0003 & 0,0000 \\
\hline
\end{tabular}


De forma a complementar os resultados obtidos, a seguir, foram analisados os resultados por porto.

\section{Porto de Salvador}

O porto de Salvador foi o que teve a maior movimentação, em 2006, na Região Nordeste, mostrando-se eficiente nos quatros cenários analisados. Os quatros cenários apresentaram folga $=0$ (zero), ou seja, o alvo foi atingido. Apresentaram pesos nulos: a variável "calado" nos cenários 2, 3 e 4 e a variável "movimentação horária" nos cenários 3 e 4 para DMU Salvador.

Dentre as variáveis de infraestrutura, que podem ser consideradas gargalos para o porto, podese destacar: profundidade, extensão e capacidade estática.

\section{Porto de Suape}

O porto foi ineficiente nos quatros cenários analisados.

A adição da variável "calado" aumentou o índice de eficiência de 0,68 para 0,92 , ou seja, a profundidade do porto ajuda a minimizar a sua ineficiência derivada das variáveis "berço" e "movimentação horária".

A análise dos alvos e folgas mostra que apenas no "cenário 1" obteve folga zero e alvo de 661 m para a variável "berço", ou seja para o porto ser eficiente bastaria ter extensão do berço de $632 \mathrm{~m}$ ao invés de $935 \mathrm{~m}$. Nos cenários 2, 3 e 4 a variável "berço" apresentou folga e o seu alvo manteve-se o mesmo do "cenário 1" $632 \mathrm{~m}$. A variável "navios" e "movimentação" apresentaram folga de 114 navios e 19.570 TEU, respectivamente. Apresentaram pesos nulos a variável "berço" nos cenários 2, 3 e 4 e a variável "navios" no "cenário 3" e “movimentação" no "cenário 4".O valor do benchmark de Suape foi de 95,65\% da DMU Salvador, nos cenários 1 e 2.

Em 2006, o porto ficou atrás apenas do porto de Salvador em termos de movimentação (em TEU), e essa diferença diminui a cada ano. No ano de 2008, foram apenas 8.500 TEU de diferença entre Salvador e Suape. 


\section{Terminal do Pecém}

O terminal do Pecém não atingiu a eficiência nos cenários analisados, em todos os cenários apresentou índice de eficiência 0,78.

Apresentaram pesos nulos a variável "calado" nos cenários 2, 3 e 4 e a variável "navios" no "cenário 3" e "movimentação" no "cenário 4". Com relação as folgas, as variáveis "berço" e "movimentação horária" apresentaram valor zero nos cenários analisados. O valor do benchmark de Pecém foi de 82,61\% da DMU Salvador, nos cenários 1 e 2.

A infraestrutura adequada encontrada no porto e a baixa movimentação de contêineres, em relação a Salvador e Suape, tornam o terminal ineficiente. Em comparação com Suape, o terminal do Pecém tem profundidade e capacidade estática igual, logo o terminal pode movimentar quantidades equivalentes ou até superiores ao porto de Suape.

\section{Porto de Fortaleza}

O porto de Fortaleza no cenário 1 ficou com índice de eficiência abaixo de 0,50 e nos demais cenários aumentou para 0,55 , sendo o porto mais ineficiente da análise.

A análise dos relatórios dos modelos apresenta pesos nulos a variável "berço" nos cenários 2 , 3 e 4 e a variável "navios" no "cenário 3" e "movimentação" no "cenário 4". Com relação as folgas, as variáveis "calado" e "movimentação horária" apresentaram valor zero nos cenários analisados. O valor do benchmark de Fortaleza foi de 47,82\% da DMU Salvador, nos cenários 1 e 2 .

A limitação do porto está na profundidade de atender navios de calado de até $10,5 \mathrm{~m}$ e a baixa movimentação horária (11 unidade/hora) por não utilizar equipamentos especializados.

\section{Porto de Natal}

Como se pode notar, o porto de Natal não foi eficiente em nenhum dos cenários.

A análise dos relatórios dos modelos apresenta pesos nulos a variável "calado" nos cenários 2, 3 e 4 e a variável "navios" no "cenários 3" e "movimentação" no "cenário 4". Com relação às folgas, as variáveis "berço" e "movimentação horária" apresentaram valor zero nos cenários 
analisados. O valor do benchmark de Natal foi de 52,17\% da DMU Salvador, nos cenários 1 e 2.

Dentre os portos analisados, é o que tem a menor participação no mercado, pois praticamente não tem capacidade de armazenamento; a profundidade do porto restringe a movimentação de navios maiores.

\section{Porto de Maceió}

O porto de Maceió não esteve na fronteira de eficiência em nenhum dos cenários analisados. Assim como Natal, Maceió não tem grande movimentação, considerando a movimentação dos outros portos analisados, tem menos de 1,5\% de participação.

A análise dos relatórios dos modelos apresenta pesos nulos a variável "calado" nos cenários 2, 3 e 4 e a variável "navios" no "cenário 3" e "movimentação" no "cenário 4". Com relação as folgas, as variáveis "berço" e "movimentação horária" apresentaram valor zero nos cenários analisados. O valor do benchmark de Maceió foi de 52,17\% da DMU Salvador, nos cenários 1 e 2 .

As instalações portuárias para as operações de contêineres são bastante limitadas. A capacidade estática de apenas 800 TEU, além de extensão de cais capaz de atender a dois navios simultaneamente.

Com a análise de cada porto apresentada, pode-se notar divisão quanto ao porte, sendo:

i. pequeno porte: Natal e Maceió, que apresentam infraestrutura precária para atender à movimentação de contêineres. Os portos necessitam melhorar a operação para movimentar mais cargas, com isso atender mais navios e aumentar a movimentação horária. Além de investimentos na operação (pessoal e equipamentos), é necessário ter instalações conservadas e apropriadas para que o porto desempenhe seu papel de maneira eficiente; 
ii. médio porte: Fortaleza, que tem baixa movimentação horária, mas apresenta movimentação significativa. Com o aumento da profundidade, poderá receber navios maiores e empregar equipamentos com alta movimentação horária, utilizando suas instalações de forma mais eficiente. Também necessita de planejamento do layout das instalações portuárias, de forma a eliminar gargalos da operação;

iii. grande porte: Salvador, Suape e Pecém, com alta movimentação horária, detêm a maior parte dos contêineres operados na região. Os portos de Suape e Pecém apresentam expectativa de serem os maiores e mais eficientes portos da região, pois os investimentos nos últimos anos são contínuos e sempre na perspectiva de atrair mais cargas. $\mathrm{O}$ porto de Salvador, que foi o único eficiente, necessita de investimentos para manter a posição, tanto nas instalações quanto na operação, além de reorganização das áreas portuárias.

\subsection{Caso de estudo 2: avaliação dos terminais de granéis sólidos}

A Tabela 9 mostra os dados dos portos que operam granéis sólidos. As variáveis de inputs são relacionadas à infraestrutura; os outputs são referentes à operação anual.

Os Estados da Bahia e do Maranhão são os que apresentam mais DMU em estudo, respectivamente, 4 e 3 . Isso evidencia que esses estados são as principais portas de escoamento de granéis sólidos da Região Nordeste.

Com os dados obtidos dos portos (DMU), a próxima etapa foi a seleção das variáveis, de modo a selecionar os dados que pudessem representar as principais características físicas e operacionais do porto, e, assim, obter a sua eficiência. Essa etapa é apresentada na seção a seguir. 
Tabela 9 - Variáveis das DMU que movimentam granéis sólidos.

\begin{tabular}{l|r|r|r|r|r|r}
\hline \multirow{2}{*}{ Variáveis } & \multicolumn{3}{|c|}{ Inputs } & \multicolumn{3}{c}{ Outputs } \\
\cline { 2 - 7 } & $\begin{array}{c}\text { Extensão } \\
\text { do berço } \\
(\mathrm{m})\end{array}$ & $\begin{array}{c}\text { Calado } \\
(\mathrm{m})\end{array}$ & $\begin{array}{c}\text { Capacidade } \\
\text { estática (t) }\end{array}$ & $\begin{array}{c}\text { Navios } \\
\text { (qde) }\end{array}$ & $\begin{array}{c}\text { Movimentação } \\
(\mathrm{t})\end{array}$ & $\begin{array}{c}\text { Movimentação } \\
\text { horária (t/dia) }\end{array}$ \\
\hline Itaqui & 474 & 11,5 & 24.840 & 73 & 1.178 .969 & 5.030 \\
\hline São Luís 1 & 1.341 & 21 & 118.000 .000 & 584 & 79.507 .243 & 85.723 \\
\hline São Luís 2 & 320 & 11 & 4.600 .000 & 115 & 4.684 .323 & 20.936 \\
\hline Fortaleza & 690 & 10,5 & 16.582 .000 & 55 & 1.193 .629 & 5.252 \\
\hline Areia Branca & 200 & 11,5 & 100.000 & 67 & 2.039 .267 & 11.309 \\
\hline Natal & 200 & 11,5 & 88.334 & 10 & 88.334 & 3.460 \\
\hline Cabedelo & 232 & 9,4 & 4.590 & 23 & 286.428 & 2.868 \\
\hline Recife & 1.338 & 9,6 & 270.500 & 136 & 1.800 .594 & 2.896 \\
\hline Suape & 386 & 14 & 160.000 & 4 & 206.316 & 6.922 \\
\hline Maceió & 750 & 11,5 & 200.000 & 136 & 2.134 .375 & 4.177 \\
\hline Inácio Barbosa & 350 & 11,5 & 2.500 .000 & 42 & 965.032 & 7.617 \\
\hline Salvador & 220 & 10 & 40.392 & 21 & 326.061 & 1.711 \\
\hline Aratu & 612 & 12 & 173.100 & 133 & 1.582 .578 & 3.082 \\
\hline Ilhéus & 432 & 11,5 & 60.000 & 23 & 711.921 & 4.191 \\
\hline Ponta da Sapoca & 230 & 11 & 60.000 & 23 & 875.865 & 5.968 \\
\hline
\end{tabular}

\section{Seleção das variáveis}

A seleção de variáveis por tipo de carga (granel sólido) adotada consistiu no uso do Método Compensatório de Normalização Única (CNU), considerada no modelo CCR.

A etapa do método de seleção Compensatório de Normalização Única (CNU) consistiu em calcular o $S$ de cada par de input-output possível.

Na primeira etapa para seleção das variáveis para granéis sólidos, foram calculados nove pares input-output. A Tabela 10 apresenta as eficiências obtidas de cada DMU, a eficiência média e os índices $S_{E F}, S_{D I S}$ e $S$ para cada par de variáveis utilizadas.

O par input-output que apresentou maior índice de eficiência média $\left(S_{E F}\right)$, da Tabela 10, foi "berço" e "navios" com valor igual a 3,66 e também apresentou o maior índice $S$, com valor de 13,662 . 
Tabela 10 - Seleção de variáveis para granéis sólidos etapa 1 com input berço.

\begin{tabular}{l|c|c|c}
\hline \multicolumn{1}{c|}{ Input } & Berço $(\mathrm{m})$ & Berço $(\mathrm{m})$ & Berço $(\mathrm{m})$ \\
\hline Itaqui & Navios & Movimentação & Movimentação horária \\
\hline São Luis 1 & 0,35 & 0,04 & 0,16 \\
\hline São Luis 2 & 1,00 & 1,00 & 0,98 \\
\hline Fortaleza & 0,83 & 0,25 & 1,00 \\
\hline Areia Branca & 0,18 & 0,03 & 0,12 \\
\hline Natal & 0,77 & 0,17 & 0,86 \\
\hline Cabedelo & 0,11 & 0,01 & 0,26 \\
\hline Recife & 0,23 & 0,02 & 0,19 \\
\hline Suape & 0,23 & 0,02 & 0,03 \\
\hline Maceió & 0,02 & 0,01 & 0,27 \\
\hline Inácio Barbosa & 0,42 & 0,05 & 0,09 \\
\hline Salvador & 0,28 & 0,05 & 0,33 \\
\hline Aratu & 0,22 & 0,02 & 0,12 \\
\hline Ilhéus & 0,50 & 0,04 & 0,08 \\
\hline Ponta da Sapoca & 0,12 & 0,03 & 0,15 \\
\hline Eficiência média & 0,23 & 0,06 & 0,40 \\
\hline$S_{E F}$ & 0,37 & 0,12 & 0,34 \\
\hline $\mathrm{N}^{\circ}$ DMU eficientes & 3,66 & 1,20 & 3,36 \\
\hline$S_{D I S}$ & 1,00 & 1,00 & 1,00 \\
\hline$S$ & 10 & 10 & 10 \\
\hline
\end{tabular}

Nesta primeira etapa, o par input-output que apresentou maior índice $S$, foi "berço" e "navios", com valor de 13,662. Além de apresentar maior índice S, também obteve o maior índice de eficiência média $\left(\mathrm{S}_{\mathrm{EF}}\right)$ igual a 3,66.

$\mathrm{Na}$ segunda etapa, adicionou-se mais uma variável, e, assim, foram calculados quatro cenários. O cenário que teve maior relação causal $\left(S_{E F}\right)$ foi aquela com adição da variável “capacidade", que resultou no valor de 6,02. Quando acrescida a variável "calado" obteve-se o maior índice $S$, com valor de 13,855 .

Na terceira etapa, foi adicionada mais uma variável, sendo gerados três cenários. O cenário que continha a variável "capacidade" obteve a maior relação causal $\left(S_{E F}\right)$, da mesma forma como na segunda etapa, porém com valor de 6,72. Quando acrescida a variável “movimentação" obteve-se o maior índice $S$ no valor de 13,855 .

Na quarta etapa, adicionou-se mais uma variável e calculou-se o índice $S$ para dois cenários. O cenário que continha a variável "capacidade" apresentou o maior valor de $S$ igual a 12,014, este cenário também foi a que apresentou maior relação causal $\left(\mathrm{S}_{\mathrm{EF}}=7,01\right)$. 
A Tabela 11 apresenta a quantidade de cenários gerados em cada etapa, as variáveis do cenário selecionado e o valor do maior índice $S$ de cada etapa.

Tabela 11 - Etapas do Método CNU para granel sólido.

\begin{tabular}{c|c|l|r}
\hline Etapas & Cenários & \multicolumn{1}{|c}{ Variáveis } & \multicolumn{2}{c}{ Índice $S$} \\
\hline 1 & 9 & $\begin{array}{l}\text { Berço } \\
\text { Navios }\end{array}$ & 13,662 \\
\hline 2 & 4 & Calado & 13,855 \\
\hline 3 & 3 & Movimentação & 13,855 \\
\hline 4 & 2 & Capacidade & 12,574 \\
\hline
\end{tabular}

Serão analisados os modelos com 2,3 e 4 variáveis, composto pelas seguintes variáveis: "berço" (input), “calado" (input), "movimentação" (output) e "navios" (output).

Não será realizado o cenário com a variável "movimentação horária", conforme o método utilizado diz que o conjunto final de variáveis a ser utilizado é o que apresentar maior índice $S$.

A etapa de seleção de variáveis é concluída com a determinação dos dados a serem utilizados na aplicação dos modelos DEA, a aplicação do modelo a ser utilizado tem que ser o mesmo modelo utilizado na seleção de variáveis.

A seção a seguir apresenta a aplicação dos modelos DEA/CCR.

\section{Análise dos resultados}

A aplicação do método DEA foi realizada por meio do SIAD - Sistema Integrado de Apoio à Decisão, desenvolvido por Angulo Meza et al. (2005).

A versão utilizada é o SIADv3.0. O programa permite utilizar até 100 DMU e 20 variáveis equilibradas entre inputs e outputs. Com a utilização do programa (SIAD), é obtida agilidade na determinação das eficiências, tendo em vista que possui interface de fácil manuseio por parte do usuário. O SIAD já foi utilizado em outros trabalhos acadêmicos, tendo propiciado resultados satisfatórios com o método DEA, o que justifica seu emprego no presente estudo.

Os granéis sólidos, carga que geralmente não possui alto valor agregado, são movimentados, principalmente, em quinze portos na Região Nordeste. 
A análise da eficiência dos portos de granel sólido foi feita com modelo CCR orientado a input. Os cenários obtidos foram de acordo com as variáveis selecionadas no método de Seleção CNU apresentado na Tabela 11.

As eficiências obtidas para a avaliação das DMU que operam granéis sólidos foram analisadas de acordo com três cenários, apresentados na Tabela 12.

Tabela 12 - Etapas do Método CNU para granel sólido.

\begin{tabular}{c|c|c|c}
\hline CENÁRIO & 1 & 2 & 3 \\
\hline & Berço & Berço & Berço \\
VARIÁVEIS & Navios & Calado & Calado \\
& & Navios & $\begin{array}{c}\text { Navios } \\
\text { Movimentação }\end{array}$ \\
\hline
\end{tabular}

Os resultados das eficiências obtidas com o modelo DEA/CCR para os cenários analisados constam na Tabela 13.

A análise dos modelos da Tabela 13 mostra que em todos os cenários analisados, os valores foram iguais, com exceção da DMU Recife no "cenário 1". Além disso, apenas a DMU "São Luís 1" foi eficiente nos cenários analisados.

Os índices dos portos ineficientes são na maioria abaixo de 0,5 , ou seja, em relação a "São Luís 1" são muito ineficientes. O porto mais ineficiente, nestes cenários, foi Suape com índice de 0,02 , praticamente nulo.

A verificação dos valores dos pesos fornecidos pelo modelo a cada variável também é importante, pois é possível analisar quais variáveis são importantes ou desconsideras pelo modelo. 
Tabela 13 - Eficiência dos portos de granéis sólidos.

\begin{tabular}{l|c|c|c}
\hline & Cenário 1 & Cenário 2 & Cenário 3 \\
\hline Itaqui & 0,35 & 0,35 & 0,35 \\
\hline São Luís 1 & 1,00 & 1,00 & 1,00 \\
\hline São Luís 2 & 0,83 & 0,83 & 0,83 \\
\hline Fortaleza & 0,18 & 0,19 & 0,19 \\
\hline Areia Branca & 0,77 & 0,77 & 0,77 \\
\hline Natal & 0,11 & 0,11 & 0,11 \\
\hline Cabedelo & 0,23 & 0,23 & 0,23 \\
\hline Recife & 0,23 & 0,51 & 0,51 \\
\hline Suape & 0,02 & 0,02 & 0,02 \\
\hline Maceió & 0,42 & 0,43 & 0,43 \\
\hline Inácio Barbosa & 0,28 & 0,28 & 0,28 \\
\hline Salvador & 0,22 & 0,22 & 0,22 \\
\hline Aratu & 0,50 & 0,50 & 0,50 \\
\hline Ilhéus & 0,12 & 0,12 & 0,12 \\
\hline Ponta da Sapoca & 0,23 & 0,23 & 0,23 \\
\hline
\end{tabular}

A Tabela 14 apresenta a média dos pesos das variáveis dos modelos DEA CCR orientado a input, os valores dos pesos por DMU.

Tabela 14 - Média dos pesos das variáveis dos modelos DEA/CCR orientado a inputs.

\begin{tabular}{l|c|c|c}
\hline \multicolumn{1}{c|}{ VARIÁVEIS } & Cenário 1 & Cenário 2 & Cenário 3 \\
\hline Berço & 0,0028 & 0,0025 & 0,0025 \\
\hline Navios & 0,0064 & 0,0066 & 0,0065 \\
\hline Calado & - & 0,0223 & 0,0223 \\
\hline Movimentação & - & - & 0,0000 \\
\hline
\end{tabular}

A variável que teve maior importância na obtenção do índice de eficiência foi "calado", seguida da variável "navios". A variável "movimentação" apresentou valor praticamente nulo, ou seja, não foi considerada no modelo para obtenção da eficiência.

As eficiências obtidas serão analisadas, a seguir, por porto. Serão também consideradas as variáveis não utilizadas na análise, a fim de propor medidas para minimizar as deficiências encontradas. 


\section{Itaqui}

O porto de Itaqui foi ineficiente em todos os cenários, apresentou índice de eficiência de 0,35.

A análise dos relatórios dos modelos apresenta pesos nulos a variável "calado" nos cenários 2 e 3 e a variável "movimentação" no cenário 3. Com relação às folgas, as variáveis "berço" e "navios" apresentaram valor zero nos cenários analisados. O valor do benchmark de Itaqui foi de 12,50 \% da DMU “São Luís 1”, nos cenários analisados.

\section{São Luís 1}

O porto de "São Luís 1" foi o único a ser eficiente em todas as análises, o que mostra tratar-se de um porto especializado e eficiente. As variáveis operacionais do porto são superiores aos demais. A movimentação horária de 85.000 t/dia é quatro vezes maior do que a segunda melhor, que é 20.900 t/dia do porto de "São Luís 2".

A análise dos relatórios dos modelos apresenta pesos nulos a variável "berço" nos cenários 2 , 3 e 4 e a variável "navios" nos cenários 3 e 4 e "movimentação horária" no "cenário 4". Com relação as folgas, todas as variáveis apresentaram valor zero nos cenários analisados.

As instalações do porto também são destaque: maior calado e extensão de berço, além de capacidade de armazenamento superior aos demais portos.

\section{São Luís 2}

O porto apesar de não ter obtido a eficiência, apresentou bons índices, nos cenários analisados, obteve índice de 0,83 .

Com base nos relatórios dos resultados obtidos no método DEA apresenta pesos nulos a variável "calado" nos cenários 2 e 3 e a variável "movimentação" no cenário 3. Com relação as folgas, as variáveis "berço" e "navios" apresentaram valor zero nos cenários analisados. O valor do benchmark de "São Luís 2" foi de 19,69 \% da DMU “São Luís 1", nos cenários 1, 2 e 3 .

Os indicadores operacionais são destaques, principalmente, a alta movimentação horária de 20.900 t/dia. 


\section{Fortaleza}

O porto de Fortaleza apresentou índices de eficiência baixos, valor abaixo de 0,20 nos três cenários.

A análise dos relatórios dos modelos apresenta pesos nulos a variável "berço" nos cenários 2 e 3 e a variável "movimentação" no cenário 3. Com relação as folgas, as variáveis "calado" e "navios" nos cenários analisados e no cenário 1 a variável "berço"apresentaram valor zero. O valor do benchmark de Fortaleza foi de 9,42 \% da DMU "São Luís 1", nos cenários analisados.

A infraestrutura do porto, considerando as variáveis analisadas, não limita a atingir sua eficiência. Com essas mesmas instalações, seria possível movimentar quantidades maiores, sendo necessário melhorar os índices de movimentação horária.

\section{Areia Branca}

O porto de Areia Branca em todos cenários obteve índice de eficiência de 0,77. Apesar de ser terminal especializado na movimentação de sal, como citado anteriormente, apresenta limitações nas instalações.

A análise dos relatórios dos modelos apresenta pesos nulos a variável "calado" nos cenários 2 e 3 e a variável "movimentação" no cenário 3. Com relação às folgas, as variáveis "berço" e "navios" nos cenários analisados apresentaram valor zero. O valor do benchmark de Areia Branca foi de 11,47 \% da DMU “São Luís 1”, em todos os cenários.

O terminal atende a apenas um navio e, por estar situado em alto mar, sua capacidade de armazenamento é baixa.

\section{Natal}

A análise do porto de Natal apresentou baixos índices de eficiência em todos cenários, não ultrapassando o valor de 0,11 . 
A análise dos relatórios dos modelos apresenta pesos nulos a variável "calado" nos cenários 2 e 3 e a variável "movimentação" no cenário 3. Com relação às folgas, as variáveis "berço" e "navios" nos cenários analisados apresentaram valor zero.

As instalações portuárias são bastante precárias: pequena profundidade, extensão de cais capaz de atender a apenas um navio. A operação também não é representativa para a região: o porto movimentou 10 navios de granéis sólidos, no ano de 2006.

\section{Cabedelo}

O porto de Cabedelo nos cenários analisados apresentou índice de eficiência de 0,23.

A análise dos relatórios dos modelos apresenta pesos nulos a variável "calado" nos cenários 2 e 3 e a variável "movimentação" no cenário 3. Com relação às folgas, as variáveis "berço" e "navios" nos cenários analisados apresentaram valor zero. O valor do benchmark de Cabedelo foi de 3,94 \% da DMU "São Luís 1" nos cenários analisados.

A profundidade do porto é a menor dentre os portos analisados; tem extensão de berço para apenas um navio e quase não tem capacidade de armazenamento. Os indicadores operacionais são satisfatórios para a quantidade movimentada, mas, para a evolução do porto, seria necessário adquirir equipamentos mais eficientes e melhorar as instalações.

\section{Recife}

O porto do Recife no "cenário 1", teve índice de eficiência de 0,23 e, nos cenários 2 e 3, valor de 0,51 .

A análise dos relatórios dos modelos apresenta pesos nulos a variável "berço" nos cenários 2 e 3 e a variável "movimentação" no cenário 3. Com relação às folgas, as variáveis "calado" e "navios" apresentaram valor zero. O valor do benchmark de Recife foi de 23,29 \% da DMU “São Luís 1”, em todos os cenários. 


\section{Suape}

O porto de Suape foi ineficiente nos três cenários; tendo valor menor do que 0,1 ou o porto não tem homogeneidade com os demais ou sua operação é bastante ineficiente.

A análise dos relatórios dos modelos apresenta pesos nulos a variável "calado" nos cenários 2 e 3, a variável "movimentação" no cenário 3. Com relação às folgas, as variáveis "berço" e "navios" nos três cenários apresentaram valor zero. O valor do benchmark de Suape foi de 0,69 \% da DMU “São Luís 1”, nos cenários 1, 2 e 3.

Suape não tem características de porto especializado em granéis sólidos; isto pode ser constatado pela movimentação de apenas quatro navios no ano de 2006, $\log$ o não há instalações especializadas para granéis sólidos. Caso venha a movimentar quantidades maiores de granéis, será necessária a aquisição de equipamentos e a readequação nas instalações.

\section{Maceió}

O porto de Maceió, na análise dos três cenários foi ineficiente.

A análise dos relatórios dos modelos apresenta pesos nulos a variável "berço" nos cenários 2 e 3 e a variável "movimentação" no cenário 3. Com relação às folgas, as variáveis "berço" no "cenário 1" e "calado" e "navios" nos cenários 2 e 3 apresentaram valor zero. O valor do benchmark de Maceió foi de 23,29 \% da DMU “São Luís 1”, nos cenários analisados.

Apesar disso, o porto tem limitação de profundidade; a capacidade estática é pequena para o volume de carga operado. Esses fatores fazem com que o porto não aproveite uma das vantagens do transporte marítimo: o ganho de escala.

\section{Inácio Barbosa}

O porto de Inácio Barbosa foi ineficiente na análise de todos os cenários. Isto mostra que tem limitação de infraestrutura na profundidade e capacidade estática; com relação à operação, poderia melhorar o tempo de atendimento do navio e a quantidade de carga operada por navio. 
A análise dos relatórios dos modelos apresenta pesos nulos a variável "calado" nos cenários 2 e 3, a variável "movimentação" no cenário 3. Com relação às folgas, as variáveis "berço" e "navios" nos cenários analisados apresentaram valor zero. O valor do benchmark de Inácio Barbosa foi de 7,20 \% da DMU "São Luís 1", nos cenários analisados.

\section{Salvador}

O porto de Salvador, em todas as análises, apresenta ineficiência. Isto indica que tanto as infraestruturas do porto, quanto a operação estão inadequadas para melhor operacionalização dos graneis sólidos movimentados em suas instalações.

A análise dos relatórios dos modelos apresenta pesos nulos a variável "calado" nos cenários 2 e 3 e a variável "movimentação" no cenário 3. Com relação às folgas, as variáveis "berço" e "navios" nos cenários analisados apresentaram valor zero. O valor do benchmark de Salvador foi de 3,60 \% da DMU “São Luís 1”, nos cenários analisados.

A profundidade do porto permite atracar navios de calado de, no máximo, $10 \mathrm{~m}$ e o berço de atracação recebe apenas um navio por vez. Com essas instalações, é complicado movimentar quantidades maiores de granel sólido, pois a tendência é usar navios maiores e que sejam carregados ou descarregados com alta movimentação horária.

\section{Aratu}

Aratu foi ineficiente na análise dos três cenários.

A análise dos relatórios dos modelos apresenta pesos nulos a variável "calado" nos cenários 2 e 3 e a variável "movimentação" no cenário 3. Com relação às folgas, as variáveis "berço" e "navios" nos cenários analisados apresentaram valor zero. O valor do benchmark de Aratu foi de 22,77 \% da DMU “São Luís 1” nos cenários analisados.

O porto tem instalações compatíveis com os demais portos, apenas tem limitação na capacidade estática. Os indicadores operacionais ainda podem ser melhorados com as instalações atuais, pois o porto de Recife, com limitação de calado, movimentou quantidade de carga superior. 


\section{Ilhéus}

O porto de Ilhéus foi ineficiente em todos os modelos analisados.

A análise dos relatórios dos modelos apresenta pesos nulos a variável "calado" nos cenários 2 e 3 e a variável "movimentação" no cenário 3. Com relação às folgas, as variáveis "berço" e "navios" nos cenários analisados apresentaram valor zero. O valor do benchmark de Ilhéus foi de 3,94 \% da DMU “São Luís 1”, nos três cenários.

Ilhéus movimenta apenas granéis sólidos e, mesmo com apenas um tipo de carga, é ineficiente, pois, observa-se que possui instalações para operar quantidades maiores de carga.

\section{Ponta da Sapoca}

O porto de Ponta da Sapoca foi ineficiente nos três cenários. O porto apresenta limitações nas suas instalações, pois tem capacidade de atender a apenas um navio, profundidade capaz de atender navios de calado de $11 \mathrm{~m}$ e capacidade estática de 60.000 t.

A análise dos relatórios dos modelos apresenta pesos nulos a variável "calado" nos cenários 2 e 3, a variável "movimentação" no cenário 3. Com relação às folgas, as variáveis "berço" e "navios" nos cenários analisados apresentaram valor zero. $\mathrm{O}$ valor do benchmark de Ponta da Sapoca foi de 3,94 \% da DMU "São Luís 1", nos três cenários.

\section{Conclusões}

O estudo derivou de uma pesquisa que analisou a eficiência relativa das operações dos portos da região Nordeste, separados por tipo de carga. O objetivo do presente estudo foi medir a eficiência portuária dos maiores portos (que movimentam contêineres no país), e, assim, constatar quais necessitam de melhorias para funcionar de forma eficiente. Para consecução do objetivo estipulado, foi utilizada o método DEA.

No que se referem aos portos que operam contêineres, as variáveis que foram selecionadas para a utilização no modelo CCR foram: berço, produtividade, calado, movimentação e navios. O “cenário 3" não alterou o resultado em relação ao “cenário 2",ou seja, apesar de adicionar 1 variável o valor do índice $S$ não alterou. Em relação a relação causal, o modelo 
que apresentou a maior relação continha as variáveis: berço, movimentação horária, calado e capacidade.

O método DEA foi aplicado para 6 portos que operam contêineres, sendo considerados eficientes, nos quatros cenários analisados, apenas o Porto de Salvador. Com relação à ineficiência, o porto de Fortaleza obteve o menor índice de eficiência, no valor de 0,46, no cenário 1. A variável "navios" apresentou peso nulo, ou seja, para obtenção do índice de eficiência não foi considerada esta variável no modelo.

No que se referem aos portos que operam granéis sólidos, as variáveis que foram selecionadas para a utilização no modelo CCR foram: berço, navios, calado e movimentação. O "cenário 2" não alterou o resultado em relação ao "cenário 3",ou seja, apesar de adicionar 1 variável o valor do índice $S$ não alterou. Em relação a relação causal, o modelo que apresentou a maior relação continha as variáveis: berço, navios, calado, movimentação e capacidade.

A análise foi aplicada em 15 portos que movimentam granéis sólidos da região Nordeste do Brasil. O terminal "São Luís 1" apresentou eficiência de 100\% em todos os cenários analisados. O porto que apresentou menor índice de eficiência foi o de Suape. A variável movimentação apresentou peso nulo, ou seja, para obtenção do índice de eficiência não foi considerada.

O ponto de ineficiência da análise é a infraestrutura e equipamentos precários, de forma a não atender aos navios de contêineres e de granéis sólidos com eficiência. O aumento da profundidade do porto quando possível, através de obras de dragagem, junto com a aquisição de equipamentos mais produtivos, irá contribuir para operar navios maiores e melhorar o desempenho do porto. 


\section{Referências}

Angulo Meza, L., Biondi Neto, L., Soares de Mello, J.C.C.B. e Gomes, E. G. (2005) ISYDSIntegrated System for Decision Support (SIAD - Sistema Integrado de Apoio a Decisão): $a$ software package for data envelopment analysis model. Pesquisa Operacional, vol. 25, n. 3, pp. 493-503.

Angulo Meza, L., Soares de Mello, J.C.C.B., Gomes, E.G. e Fernandes, A.J.S. (2007) Seleção de variáveis em DEA aplicada a uma análise do mercado de energia elétrica. Investigação Operacional, vol. 27, n. 1, pp. 21-36.

Bertoloto, R.F. e Soares de Mello, J.C.C.B. (2011) Eficiência de portos e terminais privativos brasileiros com características distintas. Journal of Transport Literature, vol. 5, n. 2, pp. 4-21.

Caixeta-Filho, J. V. e Martins, R. S. (2001) Evolução histórica da gestão logística do transporte de cargas, In: Caixeta-Filho, J. V. e Martins, R. S. (eds.) Gestão Logística do Transporte de Cargas. São Paulo: Editora Atlas.

Charnes, A., Cooper, W. W. e Rhodes, E. (1978) Measuring the efficiency of decision making units. European Journal of Operational Research, vol. 2, n. 6, pp. 429-444.

Coelli, T., Rao, D.S.P. e Battese,G.E. (1999) An introduction to efficiency and productivity analysis. Boston: Kluwer Academic Publishers.

Collyer, W. O. (2008) Lei dos portos: o conselho de autoridade portuária e a busca da eficiência. São Paulo: Lex Editora.

Collyer, W. O. (2013) A importância do direito marítimo e da regulação dos transportes e portos para o desenvolvimento da logística. Journal of Transport Literature, vol. 7, n. 1, pp. 194-201.

Cook, W. D., e Zhu, J. (2005) Modeling performance measurement: applications and implementation issues in DEA. New York: Springer.

Cortez, L. C. S., Oliveira, L. R., Martins, E. F., Jesus, I. R. D. e Soares de Mello, J. C. C. B. (2013) Análise de eficiência na gestão de portos públicos brasileiros em relação ao papel das autoridades portuárias. Journal of Transport Literature, vol. 7, n. 2, pp. 78-96.

Cullinane, K., Song, D. W., Ji, P. e Wang, T. F. (2004) An application of DEA windows analysis to container port production efficiency. Review of Networks Economics, vol. 3, n. 2, 186 - 208.

Dyson, R. G., Allen, R., Camanho, A. S., Podinovski, V. V., Sarrico, C. S. e Shale, E. A. (2001) Pitfalls and protocols in DEA. European Journal of Operations Research, vol. 132, n. 2, pp. 245-259.

Estellita Lins, M. P. e Angulo-Meza, L. (2000) Análise envoltória de dados e perspectivas de integração no ambiente de apoio à decisão. Rio de Janeiro: Editora da COPPE/UFRJ.

Fontes, O. H. P. M. e Mello, J. C. C. B. S. (2006) Avaliação da eficiência portuária através de uma modelagem DEA. X Simpósio de Pesquisa Operacional e Logística da Marinha. Anais do SPOLM.

Itoh, H. (2002) Efficiency changes at major container ports in Japan: A window application of DEA. Review of Urban \& Regional Development Studies, vol. 14, n. 2, pp. 133-152.

Martinez-Budria, E., Diaz-Armas, R., Navarro-Ibanez, M. e Ravelo-Mesa, T. (1999) A study of the efficiency of Spanish port authorities using data envelopment analysis. International Journal of Transport Economics, vol. 26, n. 2, pp. 237-253.

Rios, L. R. e Maçada, A. C. G. (2006) Analysing the relative efficiency of container terminals of Mercosul using DEA. Maritime Economics and Logistics 8, vol. 8, n. 4, pp. 331-346. 
Rodrigues, P. R. A. (2007) Introdução aos sistemas de transporte no Brasil e à logística internacional. São Paulo: Editora Aduaneiras.

Roll, Y. e Hayuth, Y. (1993) Port performance comparison applying DEA. Maritime Policy and Management, vol. 20, n. 2, pp. 153-161.

Senra. L. F. A. C., Nanci. L.C., Soares de Mello, J. C. C. B. e Angulo Meza, L. (2007) Estudo sobre métodos de seleção de variáveis em DEA. Pesquisa Operacional, vol. 27, n. 2, pp. 191-207.

Serrano, M. G. e Castellano, L. T. (2003) Analisis de la eficiencia de los servicios de infraestructura em Espana: Una application al tráfico de contenedores. Anais do X Encontro de Economia Pública.

Soares de Mello, J. C. C. B., Angulo-Meza, L., Gomes, E.G., Serapião, B. P. e Estellita Lins, M. P. (2003) Análise envoltória de dados no estudo da eficiência e dos benchmarks para companhias aéreas brasileiras. Pesquisa Operacional, vol. 23, n. 2, pp. 325-345.

Soares de Mello, M. H. C., Leta, F. R., Gomes, E. G., Soares de Mello, J. C. C. B. (2004) Análise envoltória de dados para avaliação de departamentos de ensino. Avaliação e Políticas Públicas em Educação, vol. 42, n. 12, pp. 611-622.

Sousa Junior, J. N. C., Prata, B. A. e Nobre Junior, E. F. (2008) Análise da eficiência dos portos da região nordeste do Brasil baseada em análise envoltória de dados. Sistemas \& Gestão, vol. 3, n. 2, pp. 74-91.

Talluri, S. (2000) Data envelopment analysis: models and extension. American Institute for Decision Science, vol.31, n.3, pp. 8-11.

Thanassoulis, E., Boussofiane, A. e Dyson, R. G. (1996) A comparison of data envelopment analysis and ratios as tools for performance assessment. Omega: The International Journal of Management Science, vol. 24, n. 3, pp. 229-244.

Tongzon, J. (2001) Efficiency measurement of select Australian an international port using data envelopment analysis. Transportation Research Part A, vol. 35 , n. 2, pp. 107-122.

Turner, H., Windle, R. e Dresner, M. (2004) North American containerport productivity: 1984-1997. Transportation Research Part E, vol. 40, n. 4, pp. 339-356.

Uderman, S., Rocha, C. H. e Cavalcante, L. R. (2012) Modernização do sistema portuário no Brasil: uma proposta metodológica. Journal of Transport Literature, vol. 6, n. 1, pp. 221-240.

Valentine, V. F. e Gray, R. (2001) The measurement of port efficiency using data envelopment analysis. Proceedings of the 9th World Conference on Transport Research, Seoul, South Korea. 